\title{
Granular flow through vertical slots: analysis of a general formula
}

\author{
A. Medina ${ }^{1, *}$, D.A. Serrano ${ }^{2,3, * *}, A$. López-Villa $^{1}$, and $M$. Pliego $^{2}$ \\ ${ }^{1}$ ESIME Azcapotzalco, Instituto Politécnico Nacional, Av. de las Granjas 682, Col. Sta. Catarina, Azcapotzalco 02250 CDMX, \\ México. \\ ${ }^{2}$ Instituto Tecnológico de Querétaro, Av. Tecnológico s/n, Centro, 76000 Santiago de Querétaro, Qro \\ ${ }^{3}$ Facultad de Ciencias, Universidad Nacional Autónoma de México, Exterior Circuit S/N, C.U., Coyoacán, 04510 CDMX.
}

\begin{abstract}
Currently, very little is known about reliable phenomelogical correlations to estimate the gravity-driven mass flow rate, of dry non-cohesive granular material, outflowing from thin thickness slots in vertical sidewalls of rectangular silos. Here, we validate a simple and general formula that fits pretty well data published elsewhere, including the cases of vertically and horizontally elongated slots.
\end{abstract}

\section{Introduction}

When gravity-driven side discharge of grains through circular orifices occurs, it is useful to compare it to the case of the discharge of non-cohesive grains through circular orifices in flat-bottomed silos [1]. Recent experiments have shown that this comparison, in terms of the phenomenological correlations for the mass flow rate $\dot{m}$, is suitable because it is possible to establish a relationship between the flow rate through orifices in vertical or in tilted sidewalls with that valid for orifices at a horizontal bottom [2].

In his experimental study Hagen found that the discharge rate of sand, driven by gravity, obeys the relationship $\dot{m} \sim D^{5 / 2}$ [1] when $D \gg d$, where $d$ is the mean particle diameter and $D$ is the diameter of the orifice. Now, it has been well established that the Hagen's like formulas are also valid for the discharge from circular orifices at the bottom of funnels and circular standpipes [3, 4], among others cases.

In some cases it was noticed that the granular flow can be seen as occurring through an effective cross-sectional area reduced with respect to the actual area due to the effect of the grains size, in which case the corresponding formula is the Hagen-Beverloo, $\dot{m} \sim(D-k d)^{5 / 2}$, where $k$ is a dimensionless constant of order unity $[1,5]$. In the context of numerical simulations, discrete and continuum modeling of silos discharge has also validated the Hagen and Hagen-Beverloo phenomenological correlations for flat-bottomed silos [6-8].

Giving these facts, it is important to examine if similar formulas, like those available for circular orifices in sidewalls, will remain accurate in the estimation of the flow rate through rectangular exit holes also located on the sidewalls; following the results reported by Zhou et al [9] for the flow rates through slots in

*e-mail: amedinao@ipn.mx

**e-mail: arman2390@hotmail.com

A video is available at https://doi.org/10.48448/fh95-k935 vertical sidewalls, here we comprehensively analyze a simple phenomenological formula which results valid for rectangular orifices, including the cases of vertically and horizontally elongated slots.

Discharge of grains through slot-like orifices (of rectangular shape) at the bottom or at the sidewalls of containers is also a commonly studied problem due to not only its scientific interest [10] but also, it is envisaged as a case study relevant to various technological and industrial applications $[11,12]$.

In the specific case of rectangular orifices in flat-bottomed silos, nowadays there is not a universally accepted formula for the mass flow rate [13-17], however, some authors [18-21] assert that a suitable correlation can be expressed as $\dot{m} \sim A D_{H}^{1 / 2}$ (if $D \gg d$ ), where $A$ and $D_{H}$ are the cross sectional area and the hydrodynamic diameter of the orifice, respectively.

For orifices in vertical sidewalls of rectangular silos, it was recently highlighted that, apparently, there are two different regimes where the width $W$ or the length $D$ of the rectangular orifices markedly control the discharge rate correlation [9]: in a case $\dot{m} \sim D W^{3 / 2}$ when $W / D \ll$ 1 , here defined as vertically elongated slots and, the converse case, where $\dot{m} \sim W D^{3 / 2}$ if $W / D \gg 1$, now defined as the horizontally elongated slot. In this work, we will show that the formula $\dot{m} \sim A D_{H}^{1 / 2}$ is a useful phenomenological relationship for quantifying the flow rate across rectangular orifices of negligible thickness, in vertical sidewalls since it accurately describes both extreme cases of vertically or horizontally elongated orifices. It should be noted that if the orifice thickness is not small, then, more complete correlations must take into account the considerable influence of the wall thickness $[22,23]$.

In order to reach our goal, we proceed as follows. First, we establish the phenomenological correlations for grain discharge from circular and rectangular orifices at the bottom walls, afterward, and then, we determine a 
single expression for the flow rate through rectangular slots in sidewalls, which includes cases of vertically and horizontally elongated slots. Later, we show comprehensively that such a formula is general because it fits pretty well experimental data for elongated and non-elongated slots. Finally, we present the main conclusions arising from this study. In its explicit form, the Hagen formula for the flow rate of grains through circular orifices at the bottom of reservoirs is [1]

$$
\dot{m}=c \rho_{b} g^{1 / 2} D^{5 / 2},
$$

where $c$ is the dimensionless discharge coefficient which depends on the particle properties, $\rho_{b}$ is the bulk density of the granular material, and $g$ is the acceleration due to gravity. In some cases, the grain size, $d$, could affect the magnitude of the discharge rate, then the equivalent form of equation (1), that takes into account this effect is the Hagen-Beverloo formula [1,5] given by

$$
\dot{m}=c \rho_{b} g^{1 / 2}(D-k d)^{5 / 2},
$$

where $k$ is another dimensionless constant of order unity. The term $(D-k d)$ is explained by suggesting that the fall of a particle through an aperture would be hindered if it happened to be near the edge of the hole, and might not occur at all if it were closer than its radius. The overall effect would be to reduce the effective diameter of an orifice by at least one-grain diameter. For simplicity in our treatment, in the rest of this work, we will not take the grain size into account in the relationships by assuming that $D \gg d$, i.e., large orifice size respect to the grain size.

Formulas (1) and (2) also can be used as approximately valid for circular orifices in vertical sidewalls of silos, whenever the orifice has a small thickness [22, 23], which will be achieved, for instance, by bevelling the rim of the orifice in thick sidewalls or if the sidewall is itself very thin.

For central slots at flat-bottomed silos, several authors $[13,14]$ have reported that the mass flow rate through elongated slots of width $W$ and length $D$, provided $W \ll D$ obeys the formula

$$
\dot{m}=c \rho_{b} g^{1 / 2} D W^{3 / 2},
$$

here $c$ is the corresponding discharge coefficient. At the present, formula (3) is also used for discharges from quasi-2D rectangular silos [15-17].

Now, it is pertinent to inquiry if whether or not formulas like (3) will be suitable for slots in vertical, very thin, sidewalls. The rest of this work is devoted to elucidating such a question.

\section{Discharge rates through vertical slots in sidewalls}

\subsection{Circular orifices}

As mentioned earlier, the mass flow rate for circular orifices in very thin sidewalls of bins obeys approximately the same formula used in flat-bottomed silos. This was proved experimentally [22, 23] by performing the discharge of grains through vertical sidewalls of different thin thicknesses.

When the bin sidewalls are thick, the flow rate of granular solids, $\dot{m}_{v}$, discharging from circular orifices of diameter $D$, in sidewalls of thickness $w$, is given by

$$
\dot{m}_{v}=c \rho_{b} g^{1 / 2} D^{5 / 2}\left(\alpha-\theta_{r}\right),
$$

where $\alpha$ is the internal angle of the orifice defined as $\alpha=\arctan (D / w)$ and $\theta_{r}$ is the angle of repose of the granular material; formula (4) predicts that if the granular material attains the angle of repose along of the orifice thickness the granular flow will be arrested, even if the silo is brimful. Conversely, in the limit of very small thickness, it is satisfied that $\left(\alpha-\theta_{r}\right) \rightarrow\left(\pi / 2-\theta_{r}\right) \sim 1$ for different materials [23]. Thus, formula (4) is accurately approximated by

$$
\dot{m_{v}} \simeq c \rho_{b} g^{1 / 2} D^{5 / 2},
$$

which was put to the test by inspecting how closely it fitted experimental data from silo emptying experiments [23].

\subsection{Rectangular orifices and slots}

Let us take a look at the discharge rate correlations for orifices of rectangular shape of width $W$ and length (height) $D$ located in the vertical sidewalls of bins. As is depicted in Fig. 1, there are three different slot configuration: vertically elongated slots where $W \ll D$, Fig. 1(a), near squared slots where $D \sim W$, Fig. 1(b), and horizontally elongated slots, where $D \ll W$, Fig. 1(c). It has become apparent that the strong contrast between the different slot shapes strongly affects the overall flow, this is attributed to the observation that near the slot the flow changes its direction in a manner proportional to the vertical length $D$. This shift is sudden if $D$ is small or smooth if $D$ is large.

To deal with the discharge process from slots in very thin vertical sidewalls, like those depicted in Fig. 1, in their experiments with rectangular silos Davis and Foye [12] applied the relationship of the form

$$
\dot{m}_{v}=c \rho_{b} g^{1 / 2} A D_{H}^{1 / 2},
$$

where $c$ is a dimensionless constant, $A$ is the area of the aperture and $D_{H}$ is the hydraulic diameter of the flow cross section area (as usual in hydrodynamics, $D_{H}=4 A / P$, where $P$ is the perimeter [24]).

Recently, it was noticed [21] that the first proposal of formula (6) can be found in the work by Fowler and Glastonbury [18], where they experimentally studied the flow rate through orifices of different shapes in flat-bottomed silos. Later, Brown and Richards [19] and Nedderman et al [20] pointed out that formula (6) is a generic formula for non-circular orifices in discharge cases.

Davis and Foye [12] used formula (6) to fit their experimental data, mainly in discharge cases from 

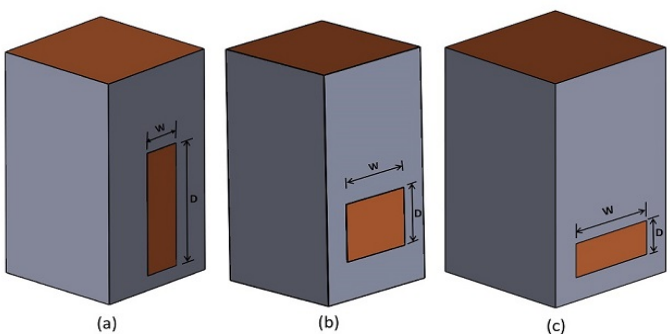

Figure 1. Sketches of bins with rectangular orifices in the vertical sidewalls: (a) vertically elongated slot $(W / D \ll 1)$, (b) near squared slot $(W / D \approx 1)$ and $(\mathrm{c})$ horizontally elongated slot $(W / D \gg 1)$.

vertically elongated slots, in the explicit form (provided $W, D \gg d)$

$$
\dot{m}_{v}=c \rho_{b} g^{1 / 2} W D\left(\frac{2 D W}{D+W}\right)^{1 / 2} \text {. }
$$

More recently, Zhou et al [9] also carried out experiments and performed numerical simulations of the discharge of grains from bins with slots in the sidewalls. They featured results for both vertically and horizontally elongated slots (without the influence of the wall thickness) and built phenomenological correlations to fit such cases.

Here, we will show that the correlation (6) (or their explicit form (7)) embraces both limit cases. A simple manner to encompass the limit cases of vertically and horizontally elongated slots is by rewriting formula (7) in terms of the ratio $W / D$, it yields the relation

$$
\dot{m_{v}}=\sqrt{2} c \rho_{b} g^{1 / 2} W D^{3 / 2} f(W / D)
$$

where

$$
f(W / D)=\left(\frac{W / D}{1+W / D}\right)^{1 / 2} .
$$

In the limit $W / D \gg 1$, the function $f(W / D)$ behaves as $f(W / D) \rightarrow 1$ and, consequently, using this result, formula (8) transforms into

$$
\dot{m}_{v} \approx \sqrt{2} c \rho_{b} g^{1 / 2} W D^{3 / 2},
$$

valid for horizontally elongated slots.

Conversely, a series expansion when $W / D \ll 1$ yields $f(W / D) \rightarrow(W / D)^{1 / 2}$, and consequently, in this limit, relationship (8) has the form

$$
\dot{m}_{v} \approx \sqrt{2} c \rho_{b} g^{1 / 2} D W^{3 / 2},
$$

corresponding to vertically elongated slots. Asymptotic relationships (10) and (11) actually represent very different limits but both of them are embraced by the single relationship (8) or, in summary, by the fundamental formula (6).

\section{Comparison to actual experimental data}

Given that the mass flow rate correlations for orifices in the sidewalls have been scarcely reported and validated, Zhou et al [9] carried out experiments of grains discharge by using non-cohesive spherical glass beads and rectangular silos, the width $W$ and length $D$ spanning the parametric region $0.1 \leq W / D \leq 14.81$; it should be noted that they beveled the lower rim of slots, along the width, to avoid the influence of the wall thickness.

In their experiments, they featured the existence of two regimes, one obeying the relationship

$$
\dot{m}_{v}=c_{W} \rho_{b} g^{1 / 2} D W^{3 / 2} \text {, if } W / D \ll 1,
$$

where $c_{W}=0.68$, which corresponds to vertically elongated slots and the other being

$$
\dot{m}_{v}=c_{D} \rho_{b} g^{1 / 2} W D^{3 / 2} \text {, if } W / D \gg 1,
$$

with $c_{D}=0.51$, corresponding to horizontally elongated slots. Both of the latest correlations confirm the existence of the aforementioned asymptotic limits (10) and (11). Zhou et al [9] also have proposed a single correlation to span their data for vertically and horizontally elongated slots by using (12) and (13), such a formula can be written as

$$
\dot{m}_{v}=\frac{c_{D} \rho_{b} g^{1 / 2} W D^{3 / 2}}{\sqrt{1+\left(\frac{c_{D}}{c_{W}}\right)^{2} D / W}},
$$

which also contains the experimental parameters $c_{W}$ and $c_{D}$ and closely fits all their data.

In order to show the validity of the correlation (7), for quantifying the mass flow across rectangular slots, experimental data from Zhou et al [9] were used. Specifically, we give the values of $W=(0.35,0.50,1.0$, $2.0,3.0,4.0)$ and $D=(0.27,0.54,1.0,1.50,2.0,2.5,3.0$, $3.5)$ used in their experiments, in summary, a set of 45 different slot geometries were obtained.

Also, it is possible to transform formulas (8) and (14) into dimensionless relationships, by rearranging each formula as follows: Eq. (8) is now

$$
\frac{\dot{m}_{v}}{\sqrt{2} \rho_{b} g^{1 / 2} W D^{3 / 2}}=c f(W / D),
$$

where $f(W / D)$ is given by (9), meanwhile Eq. (14) can be rewritten in the form

$$
\frac{\dot{m}_{v}}{\sqrt{2} \rho_{b} g^{1 / 2} W D^{3 / 2}}=\frac{c_{D}}{\sqrt{2}} \sqrt{\frac{W / D}{\left(\frac{c_{D}}{c_{W}}\right)^{2}+W / D}} .
$$

Formulas (15) (dashed curve) and (16) (symbols) are plotted in Fig. 2 where the values $c_{D}=0.51$ and $c_{W}=0.68$, as determined by Zhou et al [9] were used. We found that the best fit of formula (15) to symbols, using least-squares fit, was obtained with $c=0.37$, i.e., in this case only a single fit parameter is required, additionally, the maximum deviation between formula (16) and experimental data, at $W / D=2$, reached up $5 \%$. 
Incidentally, we found that the value $c=0.37 \approx$ $c_{D} / \sqrt{2}=0.36$ and in this case, if in formula (16) we make $c_{D}=c_{W}=\sqrt{2} c$, the formula (15) is retrieved. In the making of the plot of Fig. 2, we first fixed the value of $W$, as is stated in the inset, after that, we computed the ratios $W / D$; low (large) values of $W$ correspond to vertically (horizontally) elongated slots.

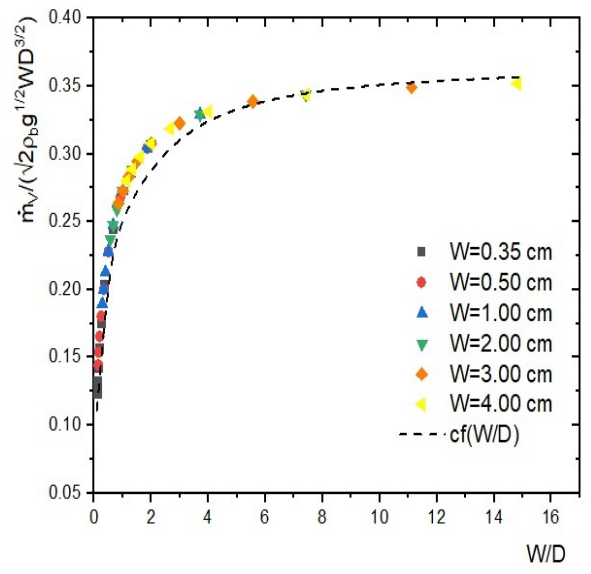

Figure 2. Dimensionless plot of Eqs. (15) (dashed curve) and (16) (symbols) as a function of $W / D$. The values of $W$ and $D$ used in Eq. (16) were given in previous paragraphs: low (large) values of $W$ correspond to vertically (horizontally) elongated slots. The best fit of formula (15) to symbols was obtained with $c=0.37$.

Based on these results, we conclude that correlation (15) satisfactorily describes the flow rates during grains discharge through any slot aspect ratio in the vertical sidewalls of silos. It also means that the fundamental formula (6) has the character of a general formula for slots, albeit it is also valid for circular orifices because in such a case the Hagen formula $\dot{m} \sim D^{5 / 2}$ will be obtained.

\section{Conclusions}

In this work, we addressed the problem of validating a general formula for the flow rate of grains discharge across rectangular orifices (slots) in sidewalls of negligible thickness. We comprehensively analyzed the formula (6) showing that it closely fits, pretty well, by evaluating a single phenomenologic parameter, the experimental data reported elsewhere [9]. Moreover, such a formula yields correctly the asymptotic expressions for cases of vertically and horizontally elongated slots, specially featured by [9]. We will remember that when the sidewall of the silo is thick, the formula (6) will be modified to include the effect of the sidewall thickness. Work along this line as well as the use of different aperture shapes is now in progress.

Acknowledgements.- AM acknowledges to CONACYT through the project "Fundamental models for the thermal methods of steam injection in EOR", which has been supported by Fondo CONACYT-SENER-HIDROCARBUROS 2015-03.

\section{References}

[1] G.H.L. Hagen, Bericht Aber die zur Bekanntmachung geeigneten Verhandlungen der Koniglich Preussischen Akademie der Wissenschaften zu Berlin, (1852)

[2] D.A. Serrano, A. Medina, G. Ruiz-Chavarria, M. Pliego and J. Klapp, Powder Tech., 286, 438(2015)

[3] R.K. Kesava and P.R. Nott, An Introduction to Granular Flow (Cambridge University Press, New York, 2008)

[4] D.A. Serrano, G. Ruiz-Chavarria, S. Alvares and A. Medina, Selected Topics of Computational and Experimental Fluid Mechanics, Environmental Science and Engineering Series, (Springer International Publishing, Switzerland, 2015)

[5] W.A. Beverloo, H.A. Leniger and J. Van de Velde, Chem. Eng. Sci., 15, 260(1961)

[6] V. Vidyapati and S. Subramaniam, Ind. Eng. Chem. Res., 52, 13171(2013)

[7] T. Tian, J. Su, J. Zhan, S. Geng and G. Xu, Particuology, 36, 127-128(2018)

[8] S. Golshan, R. Zarghami and K. Saleh, Rev. Chem. Eng. (to be published)

[9] Y. Zhow, P. Lagree, S. Popinet, P. Ruyer and P. Ausillous, J. Fluid Mech., 829, 459-485(2017).

[10] N. Zheng, H. Zhu, D. Huang and Q. Shi, Sci. Sin.Phys, Mech. Astron., 50, 090005(2020)

[11] I.I. Bagrintsev and S.S. Koshkovskii, J. Chem. Pet. Eng., 6, 503(1977)

[12] C.E. Davies and J. Foye, Trans. Inst. Chem. Engrs., 69, 369(1991)

[13] R.L. Brown and J.C. Richards, Principles of Powder Mechanics (Pergamon Press, Oxford, 1970)

[14] K. Wieghardt, Ann. Rev. Fluid Mech., 7, 89(1975)

[15] J. Choi, A. Kudrolli and M.Z. Bazant, J. Phys. Condens. Matter, 17, s2533(2005)

[16] X. Zhang, S. Zhang, G. Yang, P. Lin, Y. Tian, J.F. Wan and L. Yang, Phys. Lett. A, 380, 1301(2016)

[17] R. Maiti, G. Das and P. Kumar Das, Phys. Fluids, 29, 103303(2017)

[18] R.T. Fowler, J.R. Glastonbury, Chem. Eng. Sci., 10, 150(1959)

[19] R.L. Brown and J.C. Richards, Trans. Instn. Chem. Eng., 38, 243(1960)

[20] R.M. Nedderman, U. Tuzun, S.B. Savage and G.T. Houlsby, Chem. Eng. Sci., 37, 1597(1982)

[21] K. Saleh, S. Golshan and R. Zarghami, Chem. Eng. Sci., 192, 1011(2018)

[22] A. Medina, D. Cabrera, A. Lopez-Villa and M. Pliego, Powder Tech., 253, 270(2014)

[23] D.A. Serrano, G. Ruiz-Chavarria, M. Pliego, C.A. Vargas and A. Medina, Rev. Mex. Fís., 65, 139(2019)

[24] F.M. White, Viscous Fluid Flow (McGraw-Hill, New York, 2006) . 